\title{
interview
}

\section{Sound success}

\author{
The strong scattering of light in biological tissue impedes the development of light-based biological \\ imaging. Lihong Wang explained to Nature Photonics how the use of ultrasound can aid the deeper and \\ tighter focusing of light in scattering media.
}

\section{Why are you trying to focus light in scattering media?}

Many biological applications of light are limited to the eye because it is the only organ that is transparent. For any other organ, scattering wreaks havoc with the propagation of light. The mean free path between photon scattering events in biological tissue is of the order of $0.1 \mathrm{~mm}$ - the diameter of a human hair. When photons propagate through tissue, many random scattering events cause the light to diffuse. This 'diffusion limit' of around $1 \mathrm{~mm}$ restricts the biological applications of standard high-resolution imaging techniques such as confocal microscopy, two-photon microscopy and optical coherence tomography. We would like to be able to focus light deeply (beyond a millimetre) in tissue, but surpassing the diffusion limit and achieving high resolution is a significant challenge in many applications.

\section{How did you counteract the} scattering effects?

We use ultrasound to help us focus light deeply in biological tissue, as ultrasonic scattering is 1,000 times weaker per length than optical scattering. In our approach we focus ultrasonic waves into the region where we would like to focus light. We then illuminate the sample with light, which diffuses due to scattering. However, some of the light that interacts with the ultrasound is acousto-optically shifted in frequency. Because this modulated light comes only from the ultrasonic focus, it can be considered as a source region within the sample. When light subsequently exits the tissue we need to select the modulated light while rejecting the unmodulated light. To do this we form a hologram in a nonlinear crystal that functions as a 'mirror' for performing phase conjugation. This mirror sends the waves back to where they originated from, thereby focusing light at the ultrasonic focus. The main challenge was coming up with the idea. Although the experiment is not particularly complex, both optics and ultrasonics are involved. In particular, optical alignment and rapid switching between recording and reading the dynamic hologram are critical.

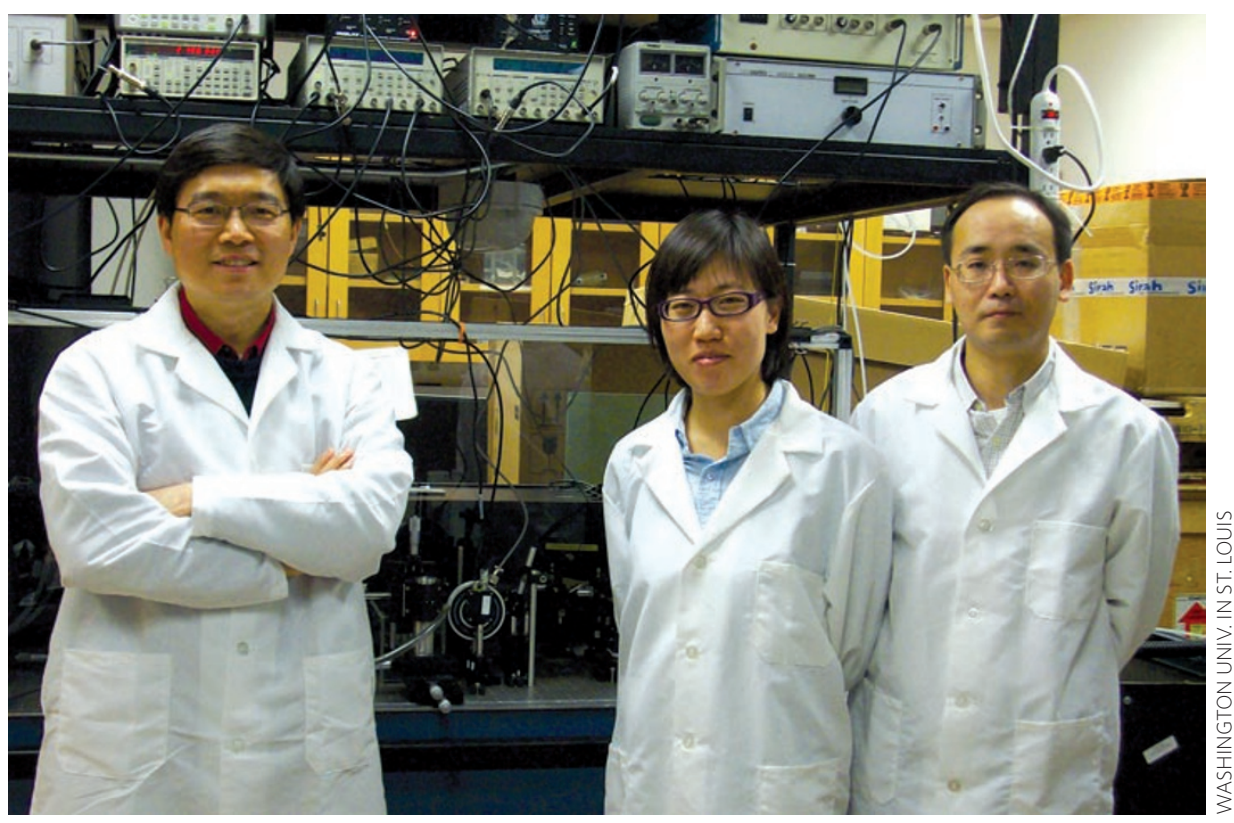

Lihong Wang (left), Honglin Liu (centre) and Xiao Xu (right) use acousto-optic modulation to aid the focusing of light in scattering media.

What applications can your ultrasoundassisted focusing be used for?

Light propagation in biological tissue has a number of important applications, including imaging, sensing, manipulation and therapy. For deep-tissue imaging and sensing, focusing light improves the spatial resolution and signal-to-noise ratio. For manipulation, focusing light improves penetration without damaging superficial tissue. For therapy, focusing light aids tumour treatment without harming the surrounding normal tissue. Our method of optical focusing could therefore potentially improve fluorescence tomography, oxymetry, optogenetics, nerve stimulation, photodynamic therapy and photothermal therapy, for example.

How tightly can the light be focused, and how can this be improved?

The optical focal spot is limited by the size of the acoustic focal spot. Right now our focal diameter is $0.8 \mathrm{~mm}$. We can increase the ultrasound frequency and shrink the focal spot, but there is a trade-off: as the frequency increases, the absorption of the ultrasonic wave increases and so the penetration depth decreases. For example, at $5 \mathrm{MHz}$ we can achieve a focal diameter of $500 \mu \mathrm{m}$ with a penetration depth of $3-6 \mathrm{~cm}$ in soft biological tissue. At $50 \mathrm{MHz}$, however, the focal diameter is reduced to around $50 \mu \mathrm{m}$, with a penetration depth of 3-6 $\mathrm{mm}$. Of course the penetration of light should be factored in as well. We have demonstrated in our lab that photo-acoustic tomography can image a target as deep as $7 \mathrm{~cm}$ in biological tissue. In the future, time-reversal gain will be a key focus for our research. Naturally, without gain the total number of photons we focus back to the focal spot can never be more than the total number emanating from it. However, the ratio of the area of diffused light in the sample to the area of the ultrasonically focused spot is at least 40 , which already indicates a significant advantage for many applications.

\section{INTERVIEW BY DAVID PILE}

Lihong Wang and colleagues have a Letter on using ultrasound to help focus light in scattering media on page 154 of this issue. 